\title{
A high-pressure gas-ionization tube calorimeter for forward detectors
}

\author{
D.M. Khazins, L. Demortier and N.D. Giokaris \\ Experimental Physics Department, The Rockefeller Universtty, New York, NY 10021-6399, USA
}

D.F. Anderson, S. Cihangir and J. Zimmerman

Fermi Natıonal Accelerator Laboratory, Batavia, IL 60510, USA

\author{
J.A. Budagov ${ }^{1}$, G.A. Ivanov, A.I. Kalinin, A.A. Kuritchin ${ }^{\dagger}$, N.O. Poroshin and S. Tokar \\ Joint Institute for Nuclear Research, Dubna, P.O. Box 79, 101000 Moscow, Russian Federation
}

G. Fanourakis and F. Lobkowicz

Department of Physlcs and Astronomy, University of Rochester, Rochester, NY 14627-0171, USA

\author{
H.R. Gustafson \\ University of Michigan, Ann Arbor, MI 48109-1120, USA
}

M. Morgan

Abulity Engineering Technology, Inc, South Holland, IL 60473, USA

Received 15 February 1993

A calorimeter design based on high-pressure gas-ionızation tubes arranged nearly parallel to the incident particle direction is described. Energy, time, and position resolutions of such a calormeter, as well as its radiation hardness and electronic noise, are discussed. Resulting characteristics meet the needs of the forward calorimetry for SSC and LHC collider experiments, and seem promising for application in other, more central parts of these experiments.

\section{Introduction}

The future SSC and LHC collider experiments have specific requirements for calorimetry in their forward (backward) regions. These calorimeters will have to work at high rates and in very intense radiation fields. The radiation dose absorbed by the SDC forward calorimeter [1], for example, will reach hundreds of Mrad per year. On the other hand the requirement on the energy resolution is not stringent. SDC requires the hadronic energy resolution to be just better than $10 \%$ at energies over $1 \mathrm{TeV}$ [1].

High-pressure noble-gas ionization calorimeters satisfy these criteria well. They are expected to be radiation hard because they have no intrinsic amplification

\footnotetext{
1 Also INFN Pisa, I-56010 Pisa, Italy.

+ Deceased.
}

and consist of radiation hard materials. The high electron mobility in commonly used gas mixtures yields an output signal comparable in width to that of a plastic scintillator counter. The lack of intrinsic amplification is also responsible for the excellent linearity and stability of these detectors. The comparatively small signal from gas-ionization calorimeters is compensated in the forward region by the high energy of the incident particles.

Work done thus far [2-8] has shown that the highpressure gas-ionization calorimetry has good energy, position and time resolution for high energy particles (starting from $E \sim 10-30 \mathrm{GeV}$ ). These detectors are very stable and have a linear energy response. Two different calorimeter designs have been explored so far, one based on parallel plates and one based on tubes. The parallel plate sandwich design was successfully used in several test calorimeters $[2,5,6,8]$. However, some difficulties such as safety, excessive dead 
space, signal broadening, and cross-talk, arise when one tries to construct a hadron calorimeter of this type with a diameter of several meters (the diameter of the SDC forward calorimeter is $\sim 3 \mathrm{~m}$ ). An attempt has been made to use gas-ionization tubes orthogonal to the incident particle direction [3]. Unfortunately it is difficult to build a tower structure in such a design, as favoured in present-day calorimeter technology. Some other proposals of gas-ionization calorimeters based on tubes [9-12] have also been made.

In this paper we discuss the design of a high-pressure gas-ionization calorimeter proposed for the forward region of SDC. This calorimeter consists of tube ionization chambers arranged nearly parallel to the incident particle direction (tilt angle $\alpha=0.8-5.7^{\circ}$ ) to allow a tower structure and to avoid dead zones formed by the tube end plugs. The main advantage of this design over the parallel plate one is safety. Besides, there are excellent signal propagation conditions in tubes, which are practically ideal waveguides. In a multi-plate structure, complex signal propagation leads to signal broadening $[13,14]$ and to cross-talk between neighboring towers.

The nonuniform transverse structure of the tube calorimeter affects the energy resolution in a different way than the sampling of parallel plate calorimeters. Of special concern is a geometry dependent constant term in the energy resolution formula, which is absent for parallel plate calorimeters. Extensive GEANT [15]
Monte Carlo simulations (described partly in refs. $[16,17])$ show a strong dependence of the tube calorimeter energy resolution on its geometrical parameters, on the type of absorber material used, and on the density of the readout gas. Nevertheless, with some reasonable choice of the calorimeter parameters, its energy resolution complies well with the needs of forward calorimetry at the SSC or LHC. The same is true of the calorimeter's electronic noise, position and time resolutions, rate capability, and radiation hardness. Design and engineering studies of this calorimeter also show it to be highly cost efficient.

\section{Calorimeter design}

The design of the tube ionization chamber for the SDC forward calorimeter is shown in fig. 1. It is made of steel and has a rectangular outer cross section of $12.7 \times 12.7 \mathrm{~mm}^{2}$, a length of $3 \mathrm{~m}$, and a coaxial hole of $9.6 \mathrm{~mm}$ diameter. A steel rod of $6.4 \mathrm{~mm}$ diameter at the center of the hole is brought to positive high voltage to collect the electrons produced by ionization. Insulating spacers support the rod and create a gap of $1.6 \mathrm{~mm}$. A high-voltage, high-pressure feedthrough plugs one end of the tube. A calorimeter module (fig. 2 ) is made by connecting 64 tubes to a gas manifold at the front end (facing incoming particles). The length of a module is equivalent to 14 nuclear interaction lengths.
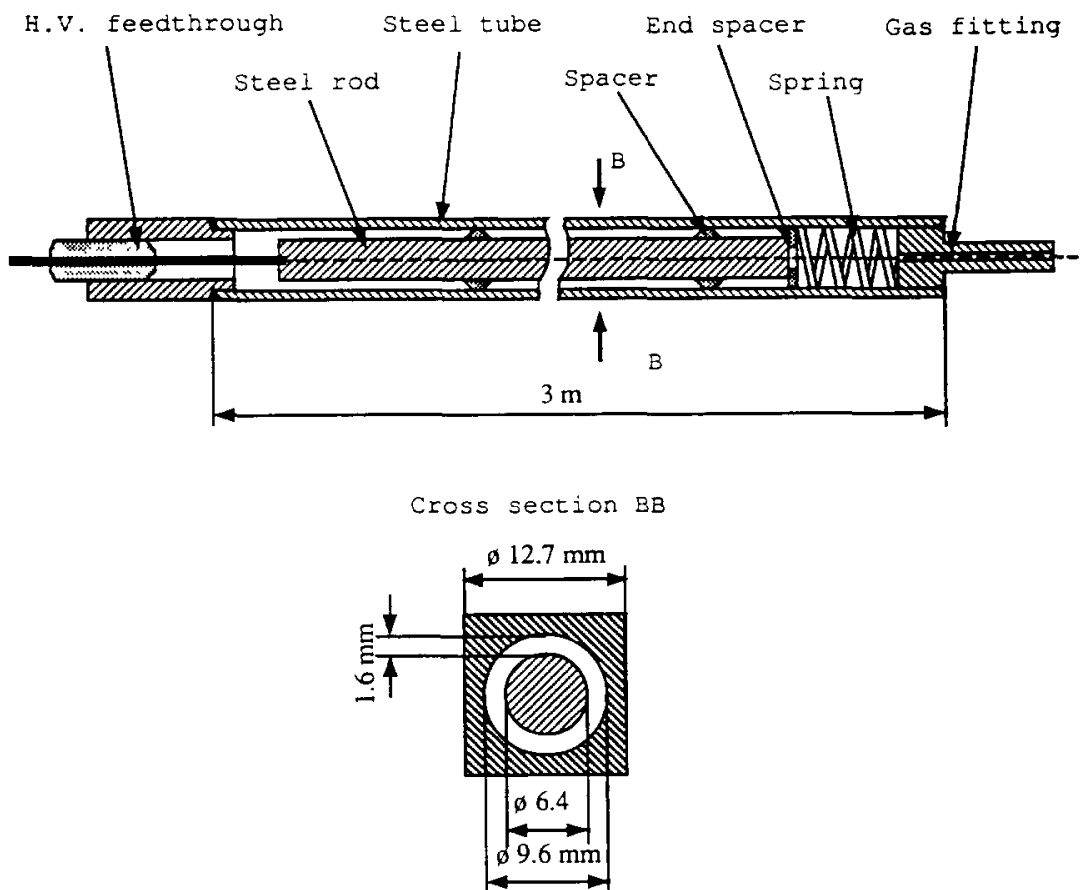

Fig. 1. Design of a tube ionization chamber. 


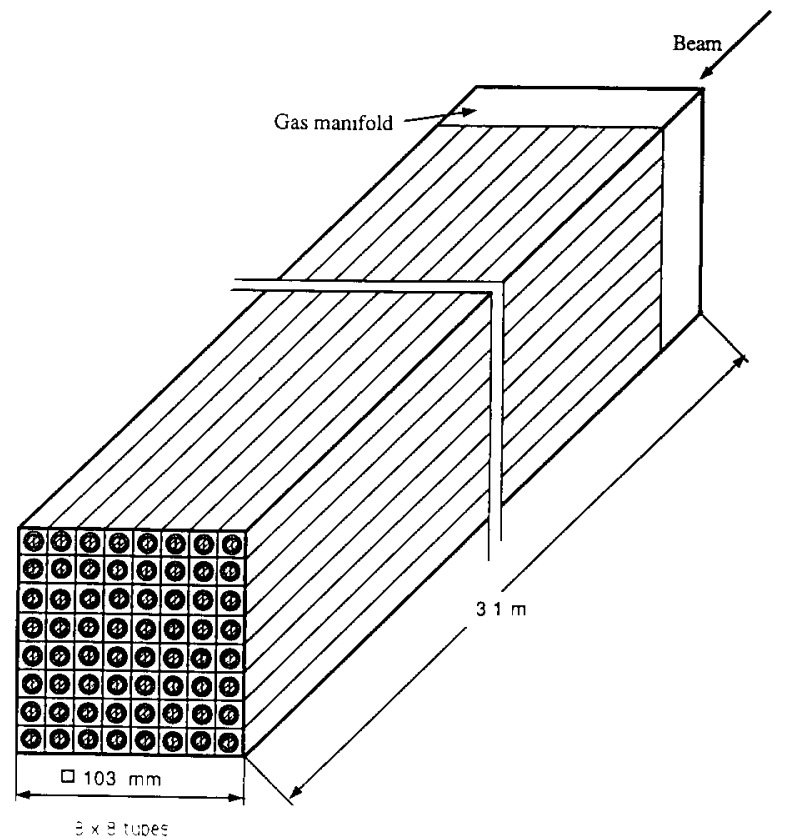

Fig. 2. Schematic of one calonmeter module.

Each module is planned to be filled with a gas mixture of $90 \% \mathrm{Ar}+10 \% \mathrm{CH}_{4}$, at a pressure of 100 atm. This is the mixture we used in the Monte Carlo simulations; however, the final choice of gas mixture will depend on results of radiation damage tests and on time resolution studies.

The SDC forward/backward calorimeter can be built from about 1600 such modules arranged parallel

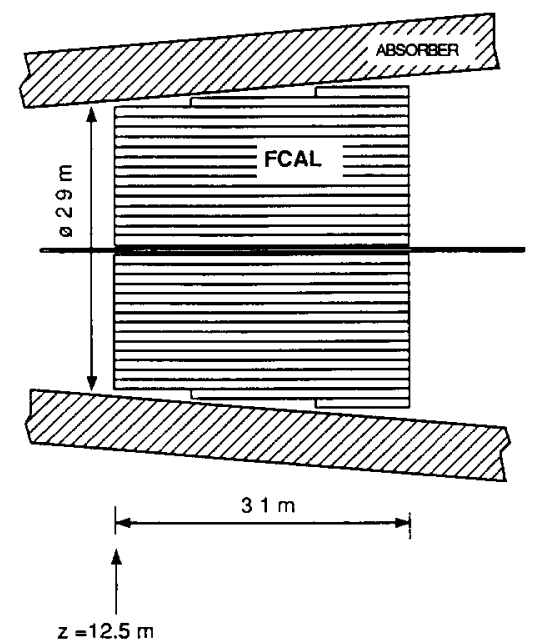

Fig. 3. Schematic diagram of the SDC forward calorimeter.
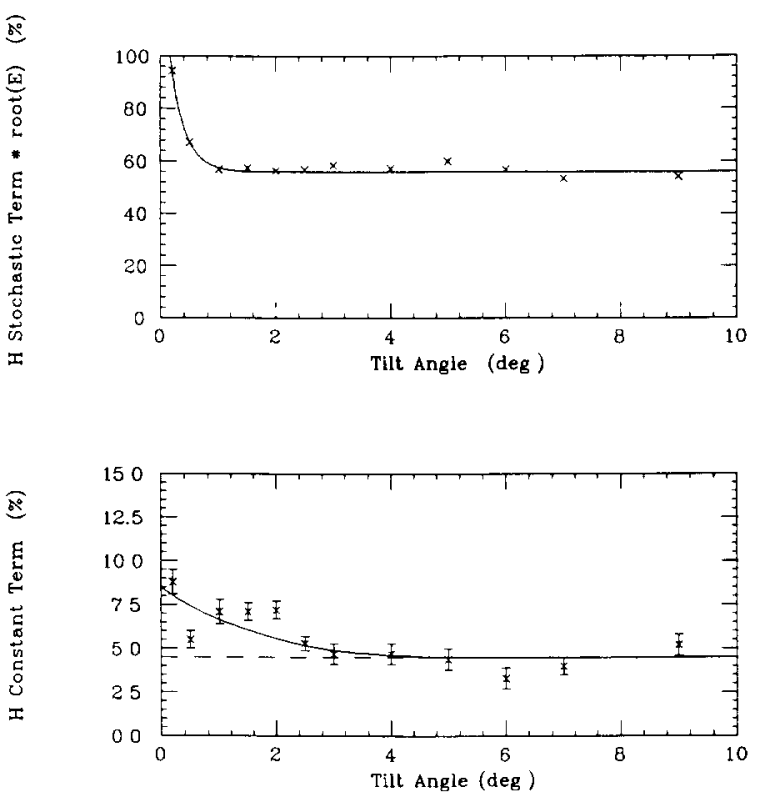

Fig. 4. The stochastic and constant terms of the hadronic (H) energy resolution, as a function of tilt angle for the tube calorimeter. The curves are to guide the eye.

to the beam direction (fig. 3). We are now constructing a prototype calorimeter based on the above design.

\section{Energy resolution}

In this section we discuss the results of Monte Carlo simulations of the calorimeter with the parameters given above. The simulated calorimeter dimensions of $3 \times 3 \times 6 \mathrm{~m}^{3}$ were chosen to exclude the effect of shower leakage. The tubes were arranged in a hexagonal structure with an absorber to gas volume ratio $n=3.08$ corresponding to that of the calorimeter design described above. The incident particle beam size was $10 \times 10 \mathrm{~cm}^{2}$, large enough to include the calorimeter nonuniformity in the energy resolution. The GEANT low-energy cut-offs were set at $0.1 \mathrm{MeV}$ for hadrons and $0.2 \mathrm{MeV}$ for electrons and photons. These cut-offs were low enough to not affect the energy resolution. The calorimeter energy resolution was studied in the range of incident particle kinetic energy $E=5-200 \mathrm{GeV}$ and was always in good agreement with the formula:

$\left(\frac{\delta E}{E}\right)^{2}=\left(\frac{A}{\sqrt{E}}\right)^{2}+B^{2}$,

where the energy $E$ is in $\mathrm{GeV}$.

The calorimeter hadronic energy resolution as a function of the tilt angle $\alpha$ is shown in fig. 4. Its stochastic coefficient $(A)$ is constant and equal to $58 \%$ 
for $\alpha>1^{\circ}$. The constant term ( $B$ ) for $\alpha>3^{\circ}$ is dominated by the inequality of the calorimeter response to hadronic and electromagnetic particles $(\mathrm{e} / \mathrm{h}=1.26)$ and is equal to $4.5 \%$, in good agreement with calculatıons by Wigmans [18]. At smaller angles the heterogeneity of the calorimeter transverse structure causes the constant term to increase with decreasing angle. The value is $\approx 7 \%$ at $\alpha=1^{\circ}$. Nevertheless, the total hadronic energy resolution of the tube calorimeter is comparatively good and meets the SDC forward calorimetry specification [1].

The electromagnetic (EM) energy resolution versus tilt angle is shown in fig. 5. The stochastic term is about $52 \% / \sqrt{E}$, which is noticeably worse than for commonly used EM calorimeters, but still meets the forward calorimetry requirements. However, the constant term is extremely large because of the narrowness of EM showers and the relatively large tubes. This results in a strong dependence of the EM signal on the position of the shower with respect to the tubes.

A possible way to reduce the EM constant term is to place a preshower converter of 3-6 radiation lengths at a small distance of $30-100 \mathrm{~cm}$ in front of the calorimeter. The converter disperses the energy of EM particles on the face of the calorimeter, thereby reduc-
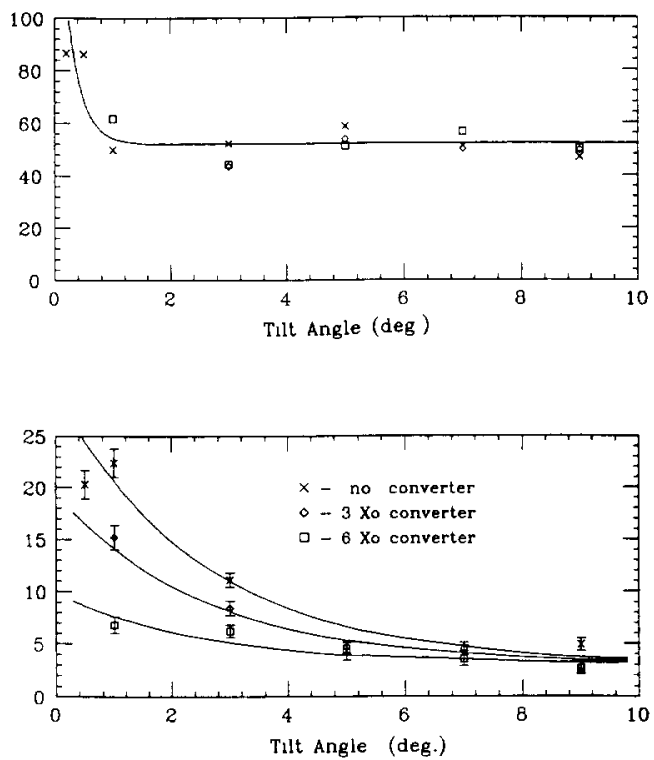

Fig. 5. The stochastic and constant terms of the electromagnetic (EM) energy resolution, as a function of tilt angle for the tube calorimeter, with and without a preshower converter at $0.5 \mathrm{~m}$ in front of the calorimeter. The curves are to guide the eye.

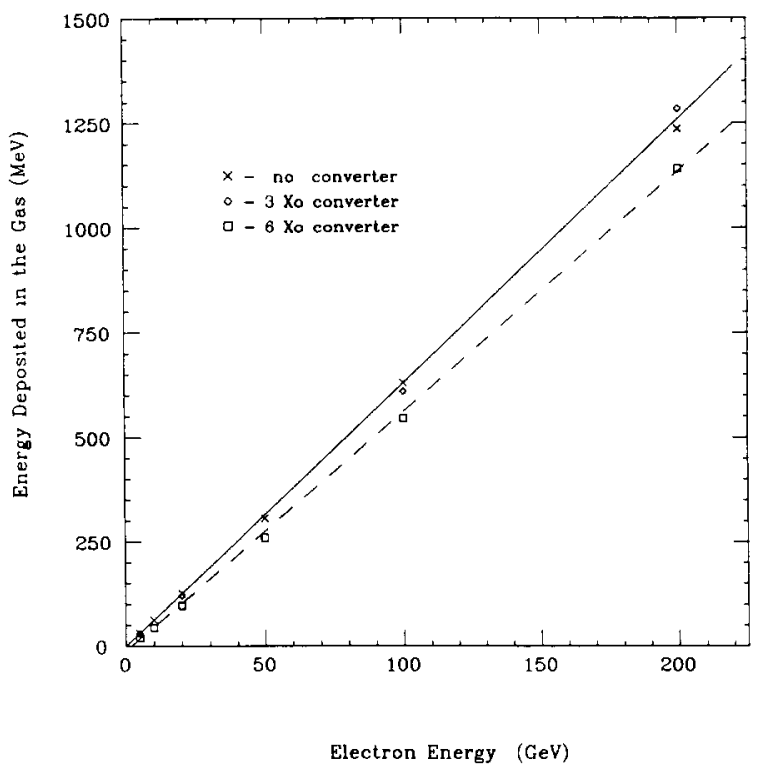

Fig. 6. Calorimeter response to electrons versus energy for three different preshower converter thıcknesses.

ing the constant term (see fig. 5). The effect of the preshower converter on the calorimeter response to electrons is shown in fig. 6 . One can see that the energy losses in the converter are not very large for high energy particles and may even be useful for a jet calorimeter (which the forward calorimeter actually is) because they partly compensate for the inequality of the calorimeter response to electrons and hadrons. A $3 X_{0}$ converter increases the EM shower width from 2.3 $\mathrm{cm}$ (rms), when it is placed immediately in front of the calorimeter, to $3.3 \mathrm{~cm}$ when it is $1 \mathrm{~m}$ away. The converter makes little effect on the hadron energy resolution.

Large amplitude fluctuations ("Texas Towers") [19], caused by scattering of slow neutrons off protons, were observed in the CDF forward hadron calorimeter, which is based on proportional chambers at normal pressure. An increase of the gas pressure proportionally increases the ionization charge released by shower particles, but makes little difference for the ionization created by short range recoil protons. Taking into account the steeply falling energy spectrum of recoil protons [19], one can expect a large suppression of "Texas Towers" with increased pressure. Our estimation gives an overall reduction in "Texas Towers" by at least a factor of $10^{7}$ compared to the CDF calorimeter.

Some examples of the simulated signal amplitude distributions are displayed in fig. 7. The spectra are slightly asymmetric at small energy; but neither asym- 
metry, nor any extra high or low energy tails, were observed at energies of $100 \mathrm{GeV}$ and higher.

\section{Electronic noise}

Electronic noise is an important parameter for gas ionization calorimeters because of their small signals. The noise for the proposed calorimeter can be calculated by considering each tube as an $\ell=3 \mathrm{~m}$ long waveguide line with impedance $Z=24 \Omega$. This tube is connected to a preamplifier with matched input impedance $\rho$ at one end, and open at the opposite end. (It is also possible to connect $n$ tubes to one preamplifier with $\rho=Z / n$.) In this case the only source of noise is the preamplifier itself, but the noise amplitude and frequency spectrum are modified by reflection of the noise signal from the open end of the line. The noise charge $Q_{1}(\mathrm{rms})$, integrated over a gate time $\tau(\tau>\ell / \nu)$, where $v$ is the wave velocity in the line, can be calcu- lated [20] as:

$Q_{1}^{2}=\left(\frac{e}{\rho}\right)^{2} \frac{\ell}{2 v}+i^{2}\left(\frac{\tau}{2}-\frac{\ell}{2 v}\right)$

Here $e$ and $l$ are the rms amplifier voltage and current noise reduced to $1 \mathrm{~Hz}$ of frequency bandwidth, respectively.

We have tested [6] two fast (rise time $\sim 10 \mathrm{~ns}$ ) and low noise amplifiers with a comparatively low input impedance $\rho=214 \Omega$ [21] and $\rho=63 \Omega$ [22]. The noise characteristics of these amplifiers are almost identical: $e \approx 0.6 \mathrm{nV} / \sqrt{\mathrm{Hz}}$ and $i \approx 3.5 \mathrm{pA} / \sqrt{\mathrm{Hz}}$. If we suppose a similar amplifier with $\rho=24 \Omega$ to be connected to each tube, and the gate time $\tau=50 \mathrm{~ns}$, then we get $Q_{1}=1.9$ fC from eq. (2).

Approximately $90 \%$ of a hadron shower occupies a volume involving about 300 tubes, resulting in an average noise $Q \sim 32 \mathrm{fC}$. This value is to be compared with the simulated calorimeter response of $13 \mathrm{fC}$ per $1 \mathrm{GeV}$ of shower energy. Thus the electronic noise produces an uncertainty $\delta \sim 2.5 \mathrm{GeV}$ in hadron energy measurements.
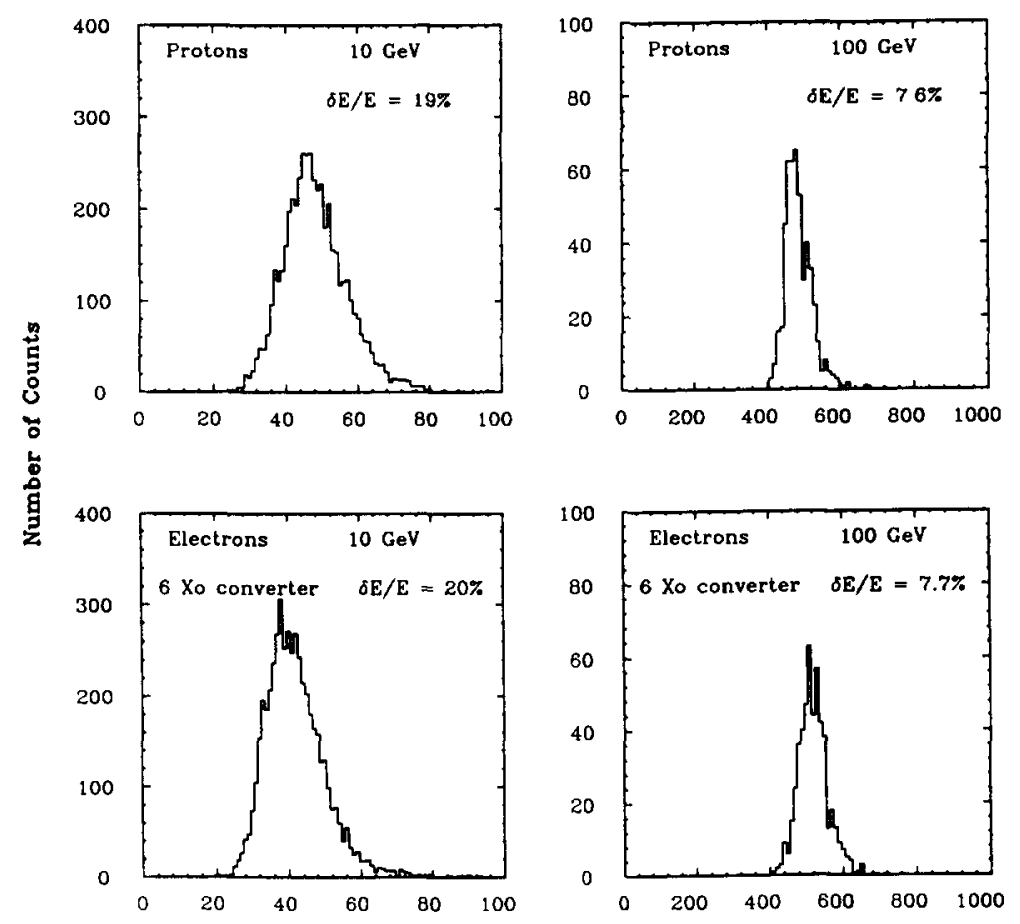

Amplitude (MeV)

Fig. 7. Examples of simulated signal amplitude distributions. Tilt angle $\alpha=3^{\circ}$. The amplitude is in units of shower energy deposited in the gas. 


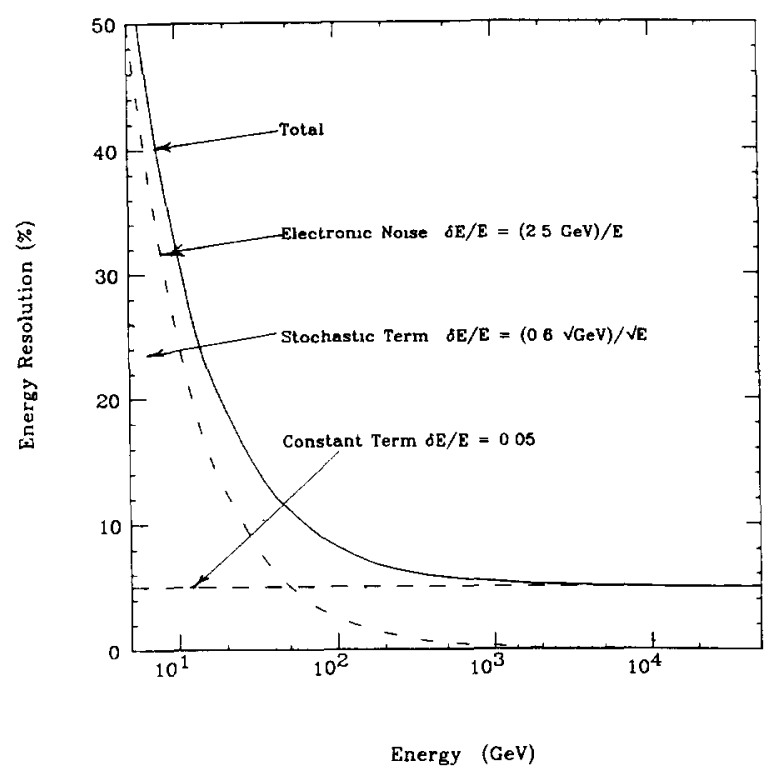

Fig. 8. Energy resolution of the tube calorimeter, as a function of particle energy.
Taking into account that the constant term for jets is somewhat smaller than for individual particles, we expect the overall SDC forward calorimeter energy resolution for jets to be:

$\left(\frac{\delta E}{E}\right)^{2}=\left(\frac{2.5}{E}\right)^{2}+\left(\frac{0.6}{\sqrt{E}}\right)^{2}=0.05^{2}$.

The dependence $\delta E / E$ on $E$ is illustrated in fig. 8 .

\section{Time resolution}

The time resolution of a tube calorimeter is limited by the time needed to collect electrons liberated in the gas. The drift velocity of electrons in mixtures of argon with $\mathrm{CH}_{4}$ or $\mathrm{CF}_{4}$ at atmospheric pressure can be as high as 5 and $10 \mathrm{~cm} / \mu$ s respectively [23]. Our measurements [24] with these mixtures do not show any appreciable dependence of the drift velocity on the gas pressure up to $100 \mathrm{~atm}$. The electron drift velocity in $95 \% \mathrm{Ar}+5 \% \mathrm{CH}_{4}$ and $97 \% \mathrm{Ar}+3 \% \mathrm{CF}_{4}$ versus the reduced electric field $(E / P)$ at various gas pressures $P$

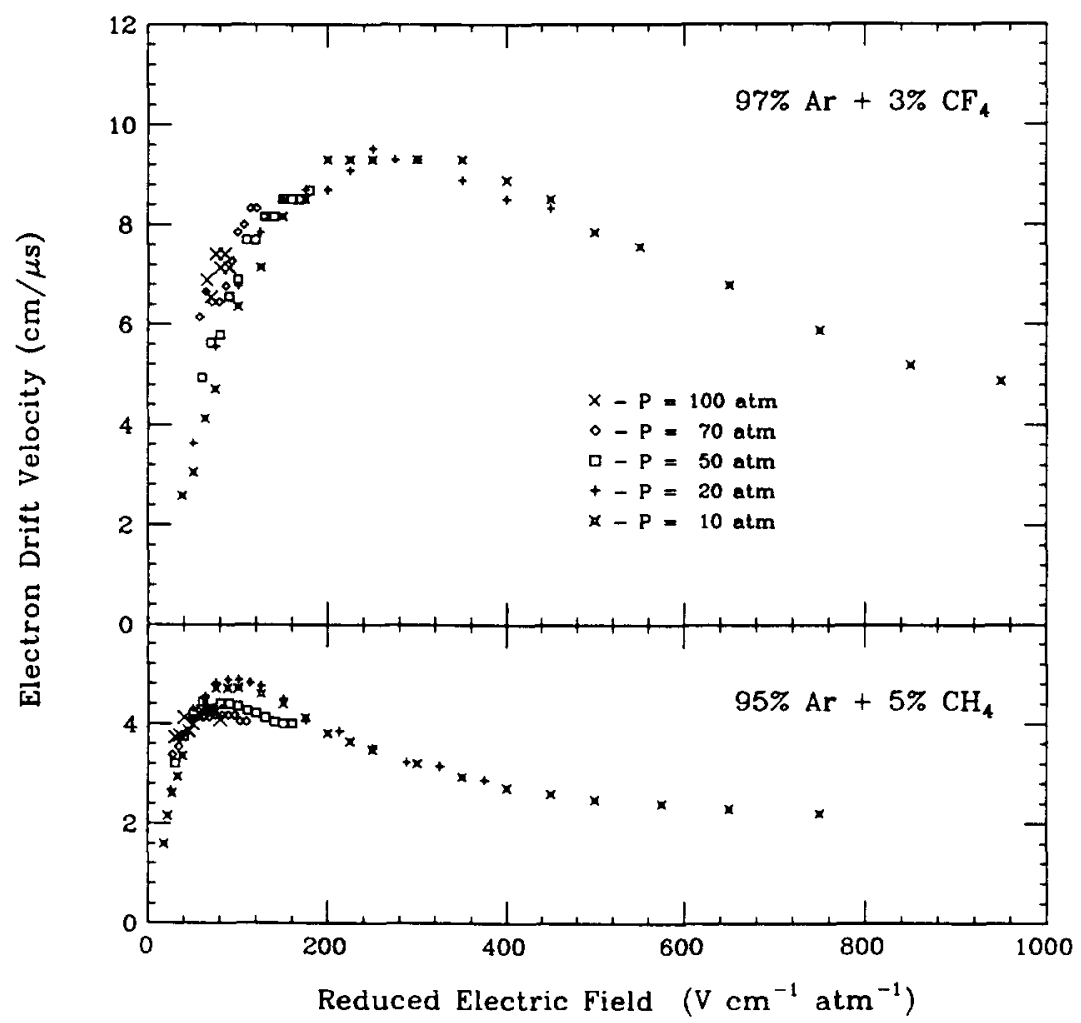

Fig. 9. Electron drift velocity in $95 \% \mathrm{Ar}+5 \% \mathrm{CH}_{4}$ and $97 \% \mathrm{Ar}+3 \% \mathrm{CF}_{4}$ gas mixtures versus the reduced electric field $(E / P)$ at various gas pressures $P$. 
is presented in fig. 9. We expect $\leq 30$ ns collection tıme for tubes with a $1.6 \mathrm{~mm}$ wide gas gap.

\section{Position resolution}

The uncertainty in the hadron shower position measurement due to lateral shower fluctuations can be smaller than $1 \mathrm{~cm}$ [25]. However, for our non-projective calorimeter arrangement (fig. 3), the longitudinal fluctuations of the shower center cause an additional uncertainty in shower positioning which can be expressed as $\delta R=\lambda \alpha$, where $R$ is the distance between the incident particle and the beam axis at the front of the forward calorimeter, and $\lambda=22 \mathrm{~cm}$ is the effective nuclear interaction length. Since in our geometry the tilt angle $\alpha=R / z$, where $z=1250 \mathrm{~cm}$ is the distance between the forward calorimeter and the nominal beam collision point, the relative position uncertainty is

$\delta R / R=0.018$.

The resulting uncertainty on the incident particle angle $\theta$ :

$\delta \theta / \theta=\delta R / R$,

contributes to the transverse energy resolution as:

$\left(\frac{\delta E_{\mathrm{t}}}{E_{\mathrm{t}}}\right)^{2}=\left(\frac{\delta \theta}{\theta}\right)^{2}+\left(\frac{\delta E}{E}\right)^{2}$.

The last term of eq. (6) dominates for our case, hence the position uncertainty does not affect the accuracy of the transverse energy measurements.

This statement is true, however, for isolated hadron and EM showers only. Both the lateral and longitudinal fluctuations of jet showers are higher, which increases the significance of the angle term in eq. (6). This problem will be investigated by Monte Carlo simulation in the future.

\section{Radiation hardness}

The radiation hardness of a gas ionization calorimeter can be estimated by comparing it with proportional chambers. By using some special gas mixtures, a proportional chamber can preserve its efficiency as long as the total charge passing through a given anode wire does not exceed $1 \mathrm{C} / \mathrm{cm}$ [26]. Such a charge will be collected by $1 \mathrm{~cm}$ of a calorimeter rod after absorbing a radiation dose of approximately 36 Mrad. However, several factors tend to suppress the polymerization and its damaging influence on ionization chamber performance. Firstly, the molecular radicals generated under irradiation are distributed uniformly in the gas volume of an ionization chamber, so that their density is much smaller than in proportional chambers, where they appear mostly in small and dense electron avalanches.
This difference is important because polymerization tends to go as radical density squared. Secondly, at the same amount of deposit the thickness of the polymer layer on the rod surface is two orders of magnitude smaller than that on an anode wire of a typical proportional chamber because of the difference in anode diameters. And lastly, the reduced electric field $(E / P)$ at the cathode surface of an ionization chamber is about ten times less than in a proportional chamber, which is crucial for spark discharge formation. As a result, one can expect the radiation hardness of a gas-lonization calorimeter to be as high as several Grads.

A radiation damage test done with a short tube similar to the one pictured in fig. 1 and filled with $95 \%$ $\mathrm{Ar}+5 \% \mathrm{CH}_{4}$ at 100 atm showed no appreciable change in the tube performance after a 50 Mrad dose of ${ }^{60} \mathrm{Co} \gamma$-ray irradiation. No deposit or whisker growth on the rod was observed. The tube was under the operating high voltage of $1.6 \mathrm{kV}$ during the irradiation. We are continuing the radiation damage studies and the results will be presented in a forthcoming publication.

\section{Space charge effect and recombination}

The problem of electric field distortion by positive ions produced by intense constant radiation in a liquid or a gas volume of an ionization chamber was investigated by Wenzel [27]. In the case of a cylindrical ionization chamber filled with a gas where the effects of electron-ion recombination and negative ion formation can be neglected, the common solution of the charge conservation equation and Poisson's equation produce the following formula for the electric field strength $E$ at radius $\xi$ :

$E^{2}=\left(\frac{2 G}{\xi}\right)^{2}+\frac{\pi I}{\mu}\left(\xi-\frac{r^{2}}{\xi}\right)^{2}$.

Here $G$ is the linear charge density of the rod of radius $r, I$ is the intensity of positive charge generation under irradiation, per unit of gas volume, and $\mu$ is the positive ion mobility.

The distortion of the electric field results in a decrease of the chamber signal amplitude because the charge induced in the amplifier by an elcctron moving inside the gap is proportional to the fraction of the potential traversed by the electron. Assuming that the field distortion is small, that the gap width $d$ is small $(d \ll r)$, and that the shower generates electrons uniformly across the gap, one can get the following approximate expression for the induced charge:

$q \approx \frac{q_{0}}{2}\left(1+\frac{d}{3 r}\right)\left\{1-\frac{\pi I d^{4}}{3 \mu V}\left(1-\frac{7 d}{15 r}\right)\right\}$, 
where $q_{0}$ is the total electron charge generated by the shower in the gap and $V$ is the chamber voltage.

For the most irradiated part of the calorimeter described above $(r=3.2 \mathrm{~mm}, d=1.6 \mathrm{~mm}, U \approx 2 \mathrm{kV}$, and $\mu=1.6 \mathrm{~cm}^{2} \mathrm{~V}^{-1} \mathrm{~s}^{-1}$ at 1 atm [28], becoming $\mu=0.016 \mathrm{~cm}^{2} \mathrm{~V}^{-1} \mathrm{~s}^{-1}$ at $\left.100 \mathrm{~atm}\right)$ the expected radiation intensity at the SSC luminosity $\left(L=10^{34} \mathrm{~cm}^{-2}\right.$ $\left.\mathrm{s}^{-1}\right)$ is $\sim 1 \mathrm{Grad} /$ year $(\sim 100 \mathrm{rad} / \mathrm{s})$. The corresponding loss of signal amplitude is $\sim 5 \%$, which is comparable with the calorimeter energy resolution and is to be taken into account for the inner most part of the forward calorimeter. The variation of electric field strength reaches $+30 \%$ at the cathode and $-10 \%$ at the anode, which could lead to a negligible increase of the signal width (see fig. 9). However, these considerations are relevant to a few inner modules of the forward calorimeter only, and there is little effect of space charge on the performance of the rest of the calorimeter because of the inverse cubic dependence of the radiation intensity on the distance from the beam axis.

An estimate of recombination based on calculations by Boag with an empirical coefficient relevant to ionion recombination in air at normal pressure and temperature [29] results in a $0.15 \%$ effect on signal amplitude at $100 \mathrm{rad} / \mathrm{s}$. Our extrapolation of this result to electron-ion recombination in $100 \mathrm{~atm}$ Ar indicates a five times higher figure, which is still too small to be of concern.

\section{Discussion}

We conclude that the high-pressure gas-ionization tube calorimeter is a fast and radiation hard detector with stable characteristics. It is also a safe, simple, easy to operate, and cost effective device. Its parameters are well within the specifications for the SDC forward calorimeter.

At the same time we believe that forward calorimetry is not the only application of this technique. The intrinsic hadronic energy resolution of the tube calorimeter described above is comparable to that used in central regions of $\mathrm{CDF}[30$ ] and $\mathrm{D} 0$ [31] detectors, or planned for SDC [1] and GEM [32], and it could be improved by refining the calorimeter structure. The only disadvantage is electronic noise, which dramatically affects the calorimeter energy resolution below 10 $\mathrm{GeV}$ and does not allow to register muons crossing the calorimeter. This disadvantage would not be so significant for future multi-TeV experiments, and it could also be reduced by further increasing the gas pressure, by electronic developments, and by refining the calorimeter structure.

The electromagnetic energy resolution of a tube calorimeter of this design is rather poor. However, this calorimeter was not optimized for EM showers. Our preliminary Monte Carlo data show that reducing the tube size by a factor of 2 proportionally decreases both the stochastic and constant terms of the EM energy resolution. At the same time, there are other, not yet investigated possibilities, such as inserting partitions in the gas gap to reduce the electron channeling along the rods, or adding some extra steel tubes inside the ionization chamber to decrease the average absorber thickness between the gas gaps. The problem of electronic noise is not as important for EM calorimeters because the volume occupied by an EM shower is much smaller than that of a hadron shower. For some of the tested EM calorimeters the noise was equivalent to $0.3 \mathrm{GeV}[5]$ and $0.7 \mathrm{GeV}[6]$.

\section{Acknowledgements}

This work was partly supported by the Texas National Research Laboratory Commission under grants nos. RGFY9160 and RGFY9260. We would like to thank Prof. J.P. Rutherfoord for interesting discussion and critical comments. We are grateful to Drs. V. Radeka and S. Rescia for useful discussions on the electronics design for the prototype tube calorimeter.

\section{References}

[1] Technical Desıgn Report of the Solenoidal Detector Collaboration, SDC-92-201 (1992).

[2] V.I. Baskakov et al., Nucl. Instr. and Meth. 159 (1979) 83.

[3] Ts. A Amatuni et al., Nucl. Instr. and Meth. 203 (1982) 179.

[4] N.D. Giokaris et al., Nucl. Instr. and Meth. A291 (1990) 552

[5] D.M. Khazins et al., Nucl. Instr. and Meth. A300 (1991) 281.

[6] L. Demortier et al., Nucl. Instr. and Meth. A324 (1993) 77.

[7] L. Demortier et al., SDC note, SDC-92-182 (1992).

[8] S. Denisov et al, III International Conference on Calorımetry in High Energy Physics, Sept. 29-Oct. 2, 1992, Corpus Christi, Texas

[9] M Barranco-Luque et al., BNL 31011, Brookhaven (1982).

[10] N.D. Giokaris et al., Proposal for the Development of High Pressure Gas Calorimetry for the SSC, submitted to SSC-DOE in June 1989 (not published).

[11] D.L. Carlsmith et al., Proposal for the Development of High Pressure Gas Calorımetry for the SSC, submitted to SSC-DOE in June 1989 (not published).

[12] E. Kistenev et al., An R\&D proposal for calorimetry in the forward direction, October 1991 (not published).

[13] V. Radeka and S. Rescia, Nucl. Instr. and Meth. A265 (1988) 228.

[14] R.N. Krasnokutsky et al., IHEP Preprint 91-16, Protvıno (1991). 
[15] R. Brun et al., CERN DD/EE/84-1 (1986).

[16] L. Demortier et al., SDC note, SDC-92-184 (1992).

[17] Yu.A. Budagov et al., JINR E1-91-572, Dubna (1991).

[18] R. Wigmans, Nucl. Instr. and Meth. A259 (1987) 389.

[19] S. Cihangir et al., IEEE Trans. Nucl. Sci. 36 (1989) 347.

[20] D.M. Khazins, FERMILAB-Pub-92/267 (1992), Nucl. Instr. and Meth. A, to be published.

[21] V.M. Golovin et al., IHEP Preprint 89-231, Protvino (1989).

[22] A.D. Volkov, JINR, Dubna (1991) (not pubished).

[23] A. Peisert and F. Sauli, CERN Preprint 84-08, Geneva (1984).

[24] N.D. Giokaris et al., FERMILAB-Pub-92/392 (1992), Nucl. Instr. and Meth. A, to be published.
[25] S. Iwata, DPNU-3-79, Nagoya (1979).

[26] J. Va'vra, Nucl. Instr, and Meth, 252 (1986) 547.

[27] W.A. Wenzel, SDC note, SDC-91-00058 (1991).

[28] E.W. McDaniel, Collision Phenomena in Ionized Gases (Wley, New York, 1964).

[29] J.W. Boag, Ionization Chambers, in: Radiation Dosimetry eds. F.H. Attix, W.C. Roesch and E. Tochilin, vol. 2 (Academic Press, New York).

[30] F. Abe et al., Nucl Instr. and Meth. A271 (1988) 387.

[31] P. Franzinı, Nucl. Instr. and Meth. A289 (1990) 438.

[32] GEM Letter of Intent, SSCL-SR-1184 and GEM TN-9249, November 30, 1991. 